\title{
RHIZOBIA AMYLASE PRODUCTION USING VARIOUS STARCHY SUBSTANCES AS CARBON SUBSTRATES
}

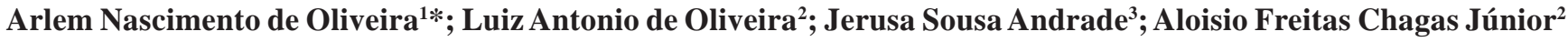 \\ ${ }^{1}$ Universidade Federal do Amazonas, Manaus, AM, Brasil; ${ }^{2}$ Coordenação de Pesquisas em Ciências Agronômicas, Instituto \\ Nacional de Pesquisas da Amazônia, Manaus, AM, Brasil; ${ }^{3}$ Coordenação de Pesquisas em Tecnologia de Alimentos, Instituto \\ Nacional de Pesquisas da Amazônia, Manaus, AM, Brasil
}

Submitted: June 07, 2006; Returned to authors for corrections: October 23, 2006 Approved: February 23, 2007.

\begin{abstract}
Six isolates of indigenous rhizobia of Central Amazonia were screened for the production of amylases in liquid media using various starchy substances as carbon sources. All rhizobia strains could produce more extracellular protein, biomass and amylases with the different kinds of carbon substrates. Among the carbon sources tested maltose was the best substrate for protein and amylase production. In general, peach palm flour and corn starch $\left(\right.$ maizena $^{\circledR}$ ) were also considered to be good carbon sources for rhizobia amylases. On the other hand, the biomass production by the rhizobia isolates was higher in the presence of oat flour. INPA strain R926 was a good amylase producer in maltose $(1.94 \mathrm{U})$ and corn starch $(0.53 \mathrm{U})$ media. INPA strain R-991 was also a good amylase producer in maltose $(1.66 \mathrm{U})$ and corn starch $(1.59 \mathrm{U})$ yielding significant extracellular amylase. Correlation analysis showed significant and positive relationships between rhizobia amylases and final $\mathrm{pH}(\mathrm{r}=0.49, \mathrm{P}<0.05)$, extracellular protein $(\mathrm{r}=0.47, \mathrm{P}<0.47)$ and biomass production $(\mathrm{r}=0.69, \mathrm{P}<0.01)$ in the maltose medium. The results obtained in this study revealed several Central Amazonian rhizobia strains as promising sources of amylase for biotechnological applications, especially in starch industry.
\end{abstract}

Key words: Rhizobium, Bradyrhizobium, cowpea, soybean, peach palm, Central Amazonia

\section{INTRODUCTION}

Amylolytic enzymes from numerous sources degrade starch, the primary storage polysaccharide in plants. Amylases are among the most important enzymes and are of great significance in present-day biotechnology, having approximately $25 \%$ of the enzyme market (30). New amylases could be potentially useful in the pharmaceutical and fine-chemical industries if enzymes with suitable properties could be identified (26). With the advent of new frontiers in biotechnology, the spectrum of amylase applications has expanded into many other fields, such as clinical, medicinal and analytical chemistry (28).

The amylases can be derived from several sources, such as plants, animals and microorganisms. Because of their short growth period, the enzymes from microbial sources generally meet industrial demands $(27,31)$. The first enzyme produced industrially was an amylase from a fungal source in 1894, which was used for the treatment of digestive disorders (7). At present, Bacillus, Aspergillus and Rhizopus species are considered to be the most important sources of industrial amylases $(14,28)$. Nevertheless, various other sources of microbial amylases are been investigated in all world.

Growth conditions and nutrients promote high yields of microbial amylases. However, carbon sources such as dextrin, fructose, glucose, lactose, maltose and starch are very expensive for commercial production of these enzymes $(14,15)$. These expensive products can be replaced in the medium with economically available agricultural by-products (11) or industrial amylaceous substances as carbon substrates. In this sense, brans, straws and flours of differente grains and tubers, such as barley, corn, cassava, potato, rice, sorghum and wheat, have been used in the fermentation medium to increase the productivity of amylases from bacteria and fungi $(2,16,29)$. Besides being the first report of occurrence of amylases in

*Corresponding Author. Mailing address: Coordenação de Pesquisas em Ciências Agronômicas - Av. André Araújo, 2936 Caixa Postal 478 - Petrópolis 69011-970 Manaus, AM - Brasil. E-mail: arlem@inpa.gov.br 
indigenous rhizobia of Central Amazonia, AM, Brazil, the present paper examines the use of alternative industrial amylaceous substances as carbon substrates for the production of amylases with rhizobial isolates.

\section{MATERIALS AND METHODS}

\section{Isolation of rhizobia, growth conditions and preliminary screening procedures}

Inoculum soil samples were mixed with sterilized sand (30:1) and placed in plastic cups $(900 \mathrm{~mL})$ in a greenhouse. Cowpea [Vigna unguiculata (L.) Walp] and soybean [Glycine max (L.) Merr.] were used in this study as trap host plants for indigenous rhizobia. Inoculum soil samples (depth of $0-10 \mathrm{~cm}$ ) were collected from rural properties of Central Amazonia, AM, Brazil. Crops, including common bean, cowpea, cassava, cabbage, pumpkin, cucumber, okra and grasses had been planted in these sites. These soils are moderately fertile, with soil $\mathrm{pH}$ around 5.5-6.5.

After 40 days nodules with pink or red-stained tissues were taken from the root system of the trap-host plants. The nodules were surface-sterilized with $95 \%$ alcohol for $10 \mathrm{~s}$, followed by a surface-sterilized with $1 \% \mathrm{NaOCl}$ for 4 min and washed several times with sterile water. The nodule contents were streaked on yeast extract-mannitol agar (YMA) medium (39) and incubated at $28^{\circ} \mathrm{C}$ in the dark. The medium contained $\left(\mathrm{g} \mathrm{L}^{-1}\right): 10.0$ mannitol, $0.4 \mathrm{~K}_{2} \mathrm{HPO}_{4}, 0.1 \mathrm{~K}_{2} \mathrm{HPO}_{4}, 0.2 \mathrm{MgSO}_{4} .7 \mathrm{H}_{2} \mathrm{O}, 0.1 \mathrm{NaCl}, 0.4$ yeast extract and 15 agar adjusted to $\mathrm{pH} 6.8$. The isolates were purified through the repeated plating method on YMA. Bacteria from single colonies were then screened for amylase production on modified YMA.

\section{Media and screening procedures}

Modified YMA medium with the substitution of the mannitol by corn starch (maizena ${ }^{\circledR}$ ) was used to detect amylolytic activity in all rhizobia strains. The plates were inoculated by transferring cells from colonies (5-7 days-old) with a flame sterilized platinum loop and were incubated for 4 days at $28^{\circ} \mathrm{C}$ in the dark. After cell growth, the Petri dishes were flooded with an iodine tinture solution. A yellow zone around the colony in an otherwise blue medium indicated starch-degrading activity (5). In this experimental condition, a total of 67 isolates were screened. The level of enzyme production was evaluated by the diameter of cleared zone (DCZ), measured in millimeters, on the reverse of the Petri dishes.

The isolates with the highest DCZ were then characterized by growth time (2-7 d incubation), colony appearance (colony colour, form and opacity), size of colony ( $\mathrm{mm}$ ), extracellular polysaccharide (EPS) production, and ability to the change $\mathrm{pH}$ of the media (acid or alkali). Indole acetic acid (IAA) production (22) in the absence of tryptophan and urea degradation (10) by the isolates were also evaluated. These isolates were then identified by partial sequencing of the $16 \mathrm{~S}$ rRNA gene at the
Center for Nuclear Energy and Agriculture (CENA, Piracicaba, $\mathrm{SP}$, Brazil). The rhizobia strains selected were investigated for production of amylases in modified yeast extract-mannitol (YM) medium (39) where the mannitol was replaced by various starchy substances as carbon substrates. YM was used as a control, and the maltose was also tested as a soluble carbon source.

\section{Effect of starchy substances on growth and amylase production}

The effect of the wheat bran, cassava, oat, peach palm, potato and tapioca flour, corn starch (maizena ${ }^{\circledR}$ ) and maltose were studied through isolate growth and amylase production after adding $1.0 \%(\mathrm{w} / \mathrm{v})$ to the modified YM medium. All amylaceous substances used in this study were purchased from the local market, Manaus, AM.

Pre-inocula were prepared in Erlenmeyer flasks, containing $30 \mathrm{~mL}$ of YM medium, previously sterilized $\left(120^{\circ} \mathrm{C}, 15 \mathrm{~min}\right)$. After cooling the medium, a loopful of bacteria was aseptically transferred to each flask. The flasks were then shaken in a rotary shaking incubator ( 65 cycles per min) at $28^{\circ} \mathrm{C}$ for 3 (fast-growing strains) or 5 days (slow-growing strains). One milliliter of the pre-inocula standardized (approximately $2.2 \times 10^{9} \mathrm{UFC} \mathrm{mL}^{-1}$ ) was added to $50 \mathrm{~mL}$ of modified YM medium and incubated in the same experimental conditions mentioned previously. Before enzymatic assay, the cells were harvested by centrifugation (12.000 rpm, $10 \mathrm{~min}$ ) and the clear supernatant was used as crude enzyme to evaluate reducing sugars, extracellular protein, amylase activity and final $\mathrm{pH}$ of the medium.

\section{Rhizobial biomass and pH determination}

At the end of cellular growth in each culture medium ( 3 and 5-days for fast and slow-growing strains, respectively), the biomass production $\left(\mathrm{g} \mathrm{L}^{-1}\right)$ was determined as cell dry weight after centrifugation ( $12.000 \mathrm{rpm}, 10 \mathrm{~min}$ ) of $2 \mathrm{~mL}$ of the culture medium in triplicate, and dried at $105^{\circ} \mathrm{C}$ overnight until constant weight. Final $\mathrm{pH}$ of the cultures was registered using a glass electrode.

\section{Analytical procedures and statistical analysis}

Amylase activity was determined by measuring the increase in reducing sugar formed by the enzymatic hydrolysis of corn starch $\left(\right.$ maizena $\left.^{\circledR}\right)$. Three hundred $\mu \mathrm{L} 1 \%$ corn starch, previously gelatinized, $0.35 \mu \mathrm{L} 0.05 \mathrm{M}$ phosphate buffer $\mathrm{pH} 6.5$ and $0.35 \mu \mathrm{L}$ enzyme supernatant were mixed and incubated at $37^{\circ}$ in a water bath for $30 \mathrm{~min}$. The final volume was adjusted to $10 \mathrm{~mL}$ with distilled water and the absorbency measured at $511 \mathrm{~nm}$ with a spectrophotometer (Spectrum UV-Vis Spectrophotometer). The amount of reducing sugar liberated was quantified by Nelson's modification of the Somogyi method (25). One enzyme unit (U) was defined as the amount of enzyme that released $1 \mu \mathrm{mol}$ of reducing sugar $\mathrm{mL}^{-1} \mathrm{~min}^{-1}$, with $\mathrm{D}$-glucose as the standard, under the standard assay conditions described above. The nonenzymatic release of sugars was corrected by setting up a 
separate blank for each sample. Extracellular protein was determined by the biuret method (13), using bovine serum albumin (BSA) as the standard curve.

Analysis of variance (ANOVA) was carried out to assess the following parameters: diameter of clearing zone, extracellular protein, production biomass and amylase activity. For the last three parameters, the design assumed cross-classification of two factors: rhizobia strains (INPA R-926, INPA R-957, INPA R975, INPA R-991, INPA R-988 and INPA R-915) and carbon substrates (wheat bran, cassava, oat, peach palm, potato and tapioca flours, corn starch, maltose and mannitol) arranged in a $6 \times 9$ factorial. All the experiments were performed in triplicate and analyzed according to Tukey's test comparisons $(\mathrm{P}<0.01)$.

Pearson product-moment correlation analysis was used to determine the relationship between amylase activity and final $\mathrm{pH}$, extracellular protein and bacterial biomass from rhizobia strains. Statistical software (StatSoft, Tulsa, USA) was used for this correlation analysis.

\section{RESULTS AND DISCUSSION}

Primary screening by starch hydrolysis on modified YMA

Sixty-seven bacterial isolates were obtained from cowpea (46) and soybean root nodules (21) of which 22 (32.8\%) exhibited amylolytic activity on modified YMA medium (Fig. 1). Nineteen amylolytic bacteria were isolated from cowpea and three from soybean nodules, respectively. Of these, bacterial isolate INPA R-926 showed the highest amylolytic activity with a DCZ of $21.4 \mathrm{~mm}$, followed by R-988, R-957, R991, R-915 and R-975 (Fig. 1).

\section{Phenotypic and genotypic characterization}

The growth time, which is often referred to as a mean characteristic to distinguish rhizobial genera (43), was estimated using incubation time (Table 1). With the exception of INPA strains R-915 and R-988, all rhizobia strains isolated from cowpea root nodules formed colonies within a time period of 2-5 days.

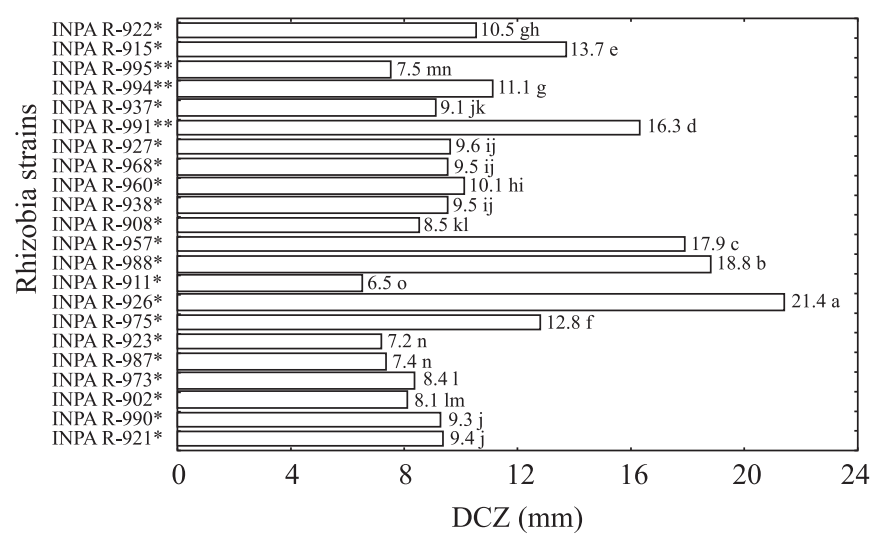

Figure 1. Diameter of clear zone (DCZ) of amylolytic rhizobia strains on modified YMA medium. $*$ and $* *=$ Rhizobia strains isolated from cowpea and soybean root nodules, respectively. Means followed by the same letter are not significantly different at $1 \%$ level.

As expected, the incubation time of colony formation of INPA strain R-991 that was isolated from soybean nodules exceeded 5 days. With respect to colony characteristics, three of the five rhizobia isolated from cowpea nodules exhibited colonies with a yellowish colour. Additionally, white colonies were produced by isolates INPA R-926, R-975 and R-991. Isolates R-991 and R988 exhibited colonies with diameters greater than $2.0 \mathrm{~mm}$. As to form and opacity of the colonies, all isolates that formed irregular colonies shaped were transparent and the circular forms were opaque. INPA strains R-926, R-991 and R-915 produced high extracellular polysaccharide slime (EPS), while the production of the other bacteria was low or moderate. Among the rhizobia strains that nodulated cowpea, only INPA R-988 was able to alkalify on YMA medium.

According to the classification of Rhizobiaceae in Bergeys's Manual (19), the two main genera of the root-nodule bacteria are

Table 1. Phenotypic characterization of isolates nodulating cowpea and soybean plants.

\begin{tabular}{|c|c|c|c|c|c|c|c|c|c|}
\hline $\begin{array}{c}\text { Rhizobia } \\
\text { strains }\end{array}$ & $\begin{array}{l}\text { Growth } \\
\text { time } \\
\text { (day) }\end{array}$ & $\begin{array}{c}\text { Colony } \\
\text { diameter } \\
\text { color }\end{array}$ & $\begin{array}{l}\text { Colony } \\
(\mathrm{mm})\end{array}$ & $\begin{array}{l}\text { Colony } \\
\text { form }\end{array}$ & $\begin{array}{c}\text { Slime } \\
\text { production }\end{array}$ & $\begin{array}{l}\text { Opacity } \\
\text { reaction }\end{array}$ & $\begin{array}{l}\text { Medium } \\
\text { production }\end{array}$ & $\begin{array}{c}\text { IAA } \\
\text { degradation }\end{array}$ & Urea \\
\hline INPAR-926* & 2 & white & $<2,0$ & irregular & high & transparent & acid & yes & yes \\
\hline INPAR-975* & $3-5$ & white & $<2,0$ & irregular & low/moderate & transparent & acid & yes & yes \\
\hline INPAR-957* & $3-5$ & yellow & $<2,0$ & circular & low/moderate & opaque & acid & yes & yes \\
\hline INPAR-991** & $5-7$ & white & $>2,0$ & circular & high & opaque & alkali & yes & yes \\
\hline INPAR-915* & $5-7$ & yellow & $<2,0$ & circular & high & opaque & acid & yes & no \\
\hline INPAR-988* & $5-7$ & yellow & $>2,0$ & irregular & low/moderate & transparent & alkali & yes & yes \\
\hline
\end{tabular}

$*$ and $* *=$ Rhizobia strains isolated from cowpea and soybean root nodules, respectively. 
Rhizobium and Bradyrhizobium. Rhizobium is characterized by fast growth on YMA, colonies that attain 2-4 $\mathrm{mm}$ in diameter within 3-5 incubation days, acid production and growth is usually accompanied by copious EPS at optimum temperature $\left(25-30^{\circ} \mathrm{C}\right)$ and $\mathrm{pH}$ of the medium (6-7). Although disparate in some characteristics, such as colonies smaller than $2.0 \mathrm{~mm}$ in diameter and low or moderate EPS production (except INPA strains R-915 and R-926), the bacterial isolates INPA R-915, R-926, R-957 and R-975 were preliminarily identified as Rhizobium. On the other hand, Bradyrhizobium is characterized by low growth, colonies do not exceed $1 \mathrm{~mm}$ within 5-7 incubation days, produce an alkaline reaction and growth is usually accompanied by EPS production under similar growth conditions to Rhizobium. Although they formed colonies larger than $1 \mathrm{~mm}$, INPA strains R-991 and R-988 were preliminarily referred to as Bradyrhizobium. Classifications based on standard phenotypic characteristics are not all embracing for indigenous rhizobia $(3,24,27)$.

In this study, IAA production was registered for all six rhizobia strains. These results agree with Boddey and Hungria (4), which found IAA production by Brazilian Bradyrhizobium
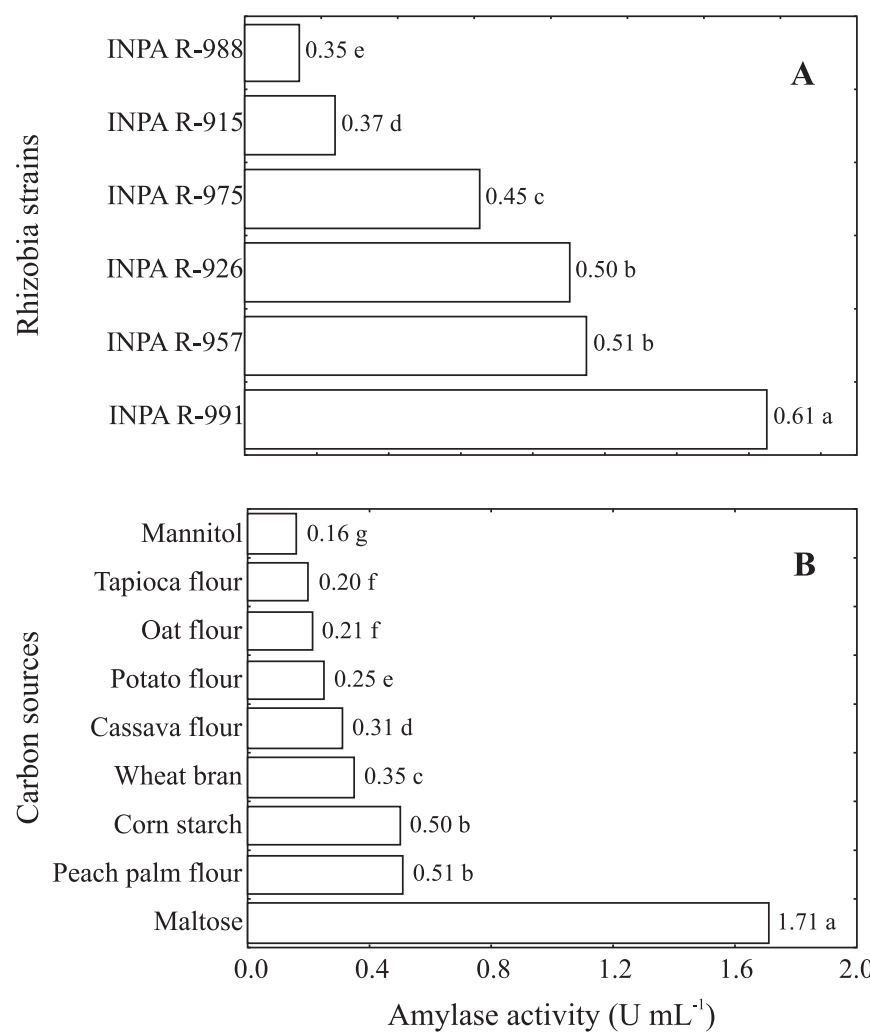

Figure 2. Amylase activity production of the rhizobia strains evaluated. (A) Averages of all nine carbon sources; (B) Averages of all six rhizobia strains. Means followed by the same letter are not significantly different at $1 \%$ level. strains nodulating soybean. As suggested by Minamisawa et al. (22) and Boddey and Hungria (4), the detection of IAA represents an easy and low-cost analysis, which shows a good relationship with species subdivision and may be used for an initial classification of rhizobia. With the exception of INPA strain R-915, all isolates were able to degrade urea. This data is in agreement with Mohamed et al. (23), who reported urea hydrolysis by various rhizobia strains isolated from Acacia spp.

The 16S rRNA sequences revealed that the bacterial isolates INPA R-915, R-926, R-957 and R-975 are closely related to Rhizobium sp. (99\% similarity), while the two other INPA strains (R-988 and 991) were closely related to Bradyrhizobium sp. (99\% similarity).

\section{Effect of different carbon substrates on the studied variables}

There were significant differences in extracellular protein, biomass and amylase activity among rhizobia strains and among carbon substrates (Figs. 2, 3 and 4), as well as a significant interaction between rhizobia strains and carbon substrates factors (Tables 2 and 3 ). All rhizobia strains could produce more
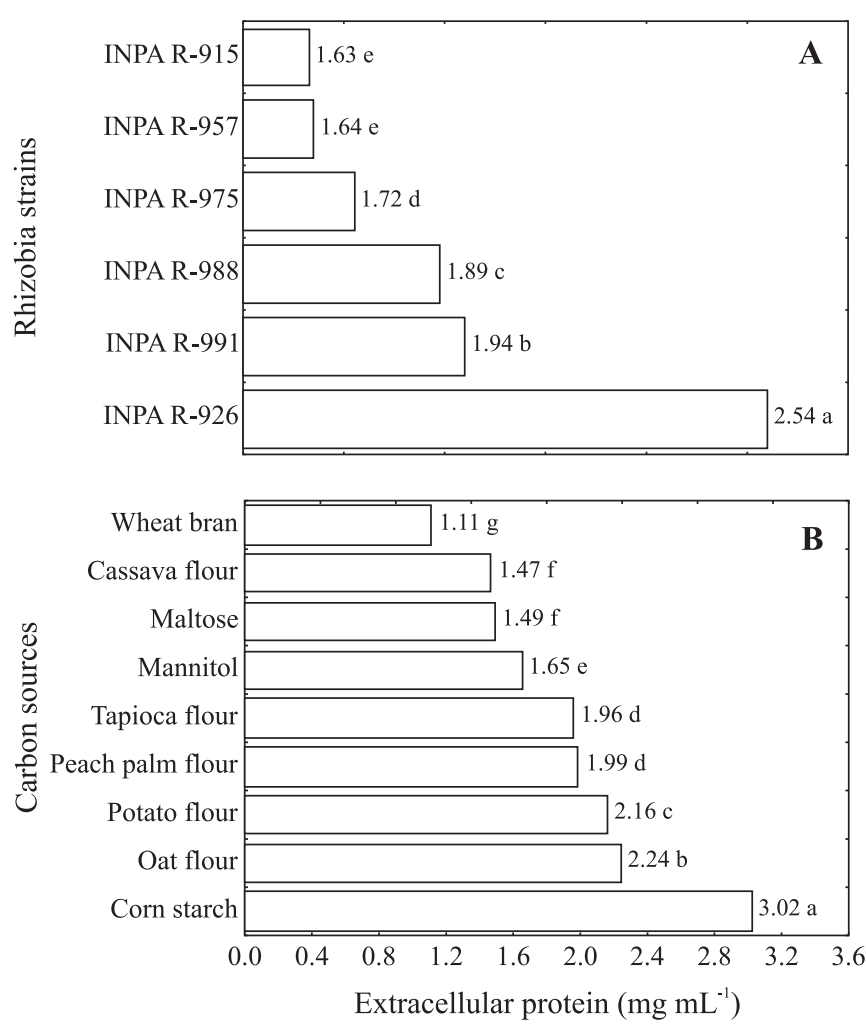

Figure 3. Extracellular protein of the rhizobia strains evaluated. (A) Averages of all nine carbon sources; (B) Averages of all six rhizobia strains. Means followed by the same letter are not significantly different at $1 \%$ level. 
Table 2. Effect of various carbon sources on extracellular protein, biomass and amylase activity production of six rhizobia strains (interactions rhizobia within carbon sources).

\begin{tabular}{|c|c|c|c|c|c|c|c|c|c|}
\hline \multirow[b]{2}{*}{$\begin{array}{l}\text { Carbon } \\
\text { substrates }\end{array}$} & \multicolumn{3}{|c|}{ INPAR-926 } & \multicolumn{3}{|c|}{ INPAR-988 } & \multicolumn{3}{|c|}{ INPAR-957 } \\
\hline & $\begin{array}{l}\text { Extracellular } \\
\text { protein } \\
\left(\mathrm{mg} \mathrm{mL}^{-1}\right)\end{array}$ & $\begin{array}{c}\text { Biomass } \\
\left(\mathrm{g} \mathrm{L}^{-1}\right)\end{array}$ & $\begin{array}{l}\text { Amylase } \\
\text { activity } \\
\left(\mathrm{UmL}^{-1}\right)\end{array}$ & $\begin{array}{l}\text { Extracellular } \\
\text { protein } \\
\left(\mathrm{mg} \mathrm{mL}^{-1}\right)\end{array}$ & $\begin{array}{c}\text { Biomass } \\
\left(\mathrm{g} \mathrm{L}^{-1}\right)\end{array}$ & $\begin{array}{l}\text { Amylase } \\
\text { activity } \\
\left(\mathrm{UmL}^{-1}\right)\end{array}$ & $\begin{array}{l}\text { Extracellular } \\
\text { protein } \\
\left(\mathrm{mg} \mathrm{mL}^{-1}\right)\end{array}$ & $\begin{array}{l}\text { Biomass } \\
\left(\mathrm{g} \mathrm{L}^{-1}\right)\end{array}$ & $\begin{array}{l}\text { Amylase } \\
\text { activity } \\
\left(\mathrm{UmL}^{-1}\right)\end{array}$ \\
\hline Wheat bran & $1.11 \mathrm{~h}$ & $0.90 \mathrm{c}$ & $0.52 \mathrm{c}$ & $1.11 \mathrm{~g}$ & $0.50 \mathrm{e}$ & $0.27 b$ & $1.07 \mathrm{~h}$ & $1.00 \mathrm{e}$ & $0.21 \mathrm{f}$ \\
\hline Cassava flour & $1.84 \mathrm{e}$ & $1.77 \mathrm{~b}$ & $0.42 \mathrm{~d}$ & $1.23 \mathrm{f}$ & $3.07 \mathrm{~b}$ & $0.19 \mathrm{c}$ & $1.26 \mathrm{~g}$ & $2.03 \mathrm{~cd}$ & $0.39 c$ \\
\hline Oat flour & $2.65 \mathrm{c}$ & $3.00 \mathrm{a}$ & $0.22 \mathrm{~g}$ & $2.45 c$ & $5.20 \mathrm{a}$ & $0.19 \mathrm{c}$ & $2.43 \mathrm{a}$ & $4.20 \mathrm{a}$ & $0.32 \mathrm{~d}$ \\
\hline Peach palm flour & $3.32 \mathrm{~b}$ & $1.70 \mathrm{~b}$ & $0.32 \mathrm{e}$ & $2.16 \mathrm{~d}$ & $3.00 \mathrm{bc}$ & $0.30 \mathrm{~b}$ & $1.50 \mathrm{e}$ & $1.93 \mathrm{~cd}$ & $1.01 \mathrm{~b}$ \\
\hline Potato flour & $2.51 \mathrm{~d}$ & $1.70 \mathrm{~b}$ & $0.24 \mathrm{f}$ & $2.83 \mathrm{~b}$ & $1.50 \mathrm{~d}$ & $0.21 \mathrm{c}$ & $1.82 \mathrm{c}$ & $3.63 \mathrm{~b}$ & $0.26 \mathrm{e}$ \\
\hline Tapioca flour & $2.52 \mathrm{~d}$ & $1.87 \mathrm{~b}$ & $0.17 \mathrm{~h}$ & $1.32 \mathrm{f}$ & $2.50 \mathrm{c}$ & $0.19 \mathrm{c}$ & $2.11 \mathrm{~b}$ & $1.50 \mathrm{de}$ & $0.21 \mathrm{f}$ \\
\hline Corn starch & $5.70 \mathrm{a}$ & $1.07 \mathrm{c}$ & $0.53 b$ & $3.41 \mathrm{a}$ & $1.17 \mathrm{~d}$ & $0.15 \mathrm{~d}$ & $1.61 \mathrm{~d}$ & $1.90 \mathrm{~cd}$ & $0.23 \mathrm{ef}$ \\
\hline Maltose & $1.69 \mathrm{f}$ & $3.20 \mathrm{a}$ & $1.94 \mathrm{a}$ & $1.26 \mathrm{f}$ & $1.60 \mathrm{~d}$ & $1.60 \mathrm{a}$ & $1.54 \mathrm{de}$ & $2.30 \mathrm{c}$ & $1.78 \mathrm{a}$ \\
\hline \multirow[t]{2}{*}{ Mannitol (Control) } & $1.49 \mathrm{~g}$ & $0.90 \mathrm{c}$ & $0.11 \mathrm{i}$ & $1.23 \mathrm{f}$ & $1.20 \mathrm{~d}$ & $0.08 \mathrm{e}$ & $1.39 \mathrm{f}$ & $1.10 \mathrm{e}$ & $0.16 \mathrm{~g}$ \\
\hline & \multicolumn{3}{|c|}{ INPAR-926 } & \multicolumn{3}{|c|}{ INPAR-988 } & \multicolumn{3}{|c|}{ INPAR-957 } \\
\hline $\begin{array}{l}\text { Carbon } \\
\text { substrates }\end{array}$ & $\begin{array}{l}\text { Extracellular } \\
\text { protein } \\
\left(\mathrm{mg} \mathrm{mL}^{-1}\right)\end{array}$ & $\begin{array}{c}\text { Biomass } \\
\left(\mathrm{g} \mathrm{L}^{-1}\right)\end{array}$ & $\begin{array}{l}\text { Amylase } \\
\text { activity } \\
\left(\mathrm{UmL}^{-1}\right)\end{array}$ & $\begin{array}{l}\text { Extracellular } \\
\text { protein } \\
\left(\mathrm{mg} \mathrm{mL}^{-1}\right)\end{array}$ & $\begin{array}{c}\text { Biomass } \\
\left(\mathrm{g} \mathrm{L}^{-1}\right)\end{array}$ & $\begin{array}{l}\text { Amylase } \\
\text { activity } \\
\left(\mathrm{UmL}^{-1}\right)\end{array}$ & $\begin{array}{l}\text { Extracellular } \\
\text { protein } \\
\left(\mathrm{mg} \mathrm{mL}^{-1}\right)\end{array}$ & $\begin{array}{l}\text { Biomass } \\
\qquad\left(\mathrm{g} \mathrm{L}^{-1}\right)\end{array}$ & $\begin{array}{l}\text { Amylase } \\
\text { activity } \\
\left(\mathrm{UmL}^{-1}\right)\end{array}$ \\
\hline Wheat bran & $1.11 \mathrm{~g}$ & $0.83 \mathrm{f}$ & $0.55 \mathrm{~b}$ & $1.02 \mathrm{e}$ & $0.53 \mathrm{i}$ & $0.27 \mathrm{~d}$ & $1.29 \mathrm{e}$ & $0.47 \mathrm{f}$ & $0.27 \mathrm{~cd}$ \\
\hline Cassava flour & $1.47 \mathrm{~d}$ & $1.70 \mathrm{c}$ & $0.30 \mathrm{~b}$ & $1.81 \mathrm{~b}$ & $2.77 \mathrm{~d}$ & $0.22 \mathrm{e}$ & $1.21 \mathrm{e}$ & $1.93 \mathrm{~d}$ & $0.35 \mathrm{c}$ \\
\hline Oat flour & $2.24 b$ & $5.00 \mathrm{a}$ & $0.16 \mathrm{~b}$ & $1.67 \mathrm{c}$ & $3.63 \mathrm{~b}$ & $0.14 \mathrm{f}$ & $2.01 \mathrm{~b}$ & $3.80 \mathrm{a}$ & $0.21 \mathrm{~d}$ \\
\hline Peach palm flour & $1.43 \mathrm{de}$ & $1.10 \mathrm{e}$ & $0.54 \mathrm{~b}$ & $1.84 \mathrm{~b}$ & $3.95 \mathrm{a}$ & $0.37 \mathrm{~b}$ & $1.67 \mathrm{c}$ & $2.30 \mathrm{~cd}$ & $0.51 \mathrm{~b}$ \\
\hline Potato flour & $2.10 \mathrm{~b}$ & $2.06 \mathrm{~b}$ & $0.23 \mathrm{~b}$ & $1.61 \mathrm{c}$ & $2.93 \mathrm{c}$ & $0.31 \mathrm{c}$ & $2.10 \mathrm{ab}$ & $3.17 \mathrm{~b}$ & $0.25 \mathrm{~d}$ \\
\hline Tapioca flour & $2.20 \mathrm{~b}$ & $1.50 \mathrm{~d}$ & $0.22 \mathrm{~b}$ & $1.43 \mathrm{~d}$ & $0.97 \mathrm{~h}$ & $0.20 \mathrm{e}$ & $2.17 \mathrm{a}$ & $2.80 \mathrm{bc}$ & $0.22 \mathrm{~d}$ \\
\hline Corn starch & $4.25 \mathrm{a}$ & $0.97 \mathrm{ef}$ & $1.59 \mathrm{a}$ & $1.67 \mathrm{c}$ & $1.70 \mathrm{e}$ & $0.13 \mathrm{e}$ & $1.50 \mathrm{~d}$ & $1.20 \mathrm{e}$ & $0.35 \mathrm{c}$ \\
\hline Maltose & $1.30 \mathrm{f}$ & $1.80 \mathrm{c}$ & $1.66 \mathrm{a}$ & $1.37 \mathrm{~d}$ & $1.10 \mathrm{~g}$ & $1.63 \mathrm{a}$ & $1.76 \mathrm{c}$ & $4.13 \mathrm{a}$ & $1.65 \mathrm{a}$ \\
\hline Mannitol (Control) & $1.36 \mathrm{ef}$ & $0.60 \mathrm{~g}$ & $0.26 \mathrm{~b}$ & $2.22 \mathrm{a}$ & $1.30 \mathrm{f}$ & $0.09 \mathrm{~g}$ & $1.75 \mathrm{c}$ & $1.90 \mathrm{~d}$ & $0.24 \mathrm{~d}$ \\
\hline
\end{tabular}

Means followed by the same letter are not significantly different at $1 \%$ level.

total extracellular protein, bacterial biomass and amylases with different kinds of carbon substrates. Rhizobium sp. strain INPA R-926, Bradyrhizobium sp. strains INPA R-988 and R-991 produced more extracellular protein using corn starch as the carbon substrate while oat flour, mannitol and tapioca flour proved to be good carbon sources for protein secretion by Rhizobium sp. strains INPA R-957, R-915 and R-975, respectively. With the exception of the INPAR-915, that exhibited a high biomass production in the peach palm medium (Table 2), the highest bacterial biomass yield was registered with oat flour. INPA strains R-926 and R-915 exhibited high biomass yield using maltose as sole carbon substrate (Table 2).

In this study, the effects of different carbon sources on biomass yield showed that the bacterial isolates behaved differently. Additionally, oat and peach palm flour could constitute cheap carbon sources for a good bacterial growth. Similar conclusions have also been obtained for other microorganisms. Ramesh et al. (29) reported that biomass production by Clostridium thermosulfurogenes using barley and tapioca flour did not differ significantly when soluble starch was used as carbon source. No significant difference was detected in biomass production by Rhodothermus marinus when maize starch (insoluble) and maltose were used as growth substrate (12). Haq et al. (16) obtained greater biomass yield by Bacillus licheniformis using pearl millet and rice than with soluble starch.

In relation to amylase activity, all the isolates were able to produce this enzyme to different extents using different carbon substrates. Comparatively, maltose was considered to be the best substrate among the tested carbon sources (Table 2 and Fig. 2b). Except for the interaction rhizobia amylase production within tapioca flour, the other interactions were significant (Table 3). INPA strain R-926 was a good amylase producer in the 
Table 3. Effect of various carbon sources on extracellular protein, biomass and amylase activity production of six rhizobia strains (interactions carbon sources within rhizobia strains).

\begin{tabular}{|c|c|c|c|c|c|c|c|c|c|}
\hline \multirow[b]{2}{*}{$\begin{array}{l}\text { Rhizobia } \\
\text { strans }\end{array}$} & \multicolumn{3}{|c|}{ Mannitol (Control) } & \multicolumn{3}{|c|}{ Peach palm flour } & \multicolumn{3}{|c|}{ Cassava flour } \\
\hline & $\begin{array}{l}\text { Extracellular } \\
\text { protein } \\
\left(\mathrm{mg} \mathrm{mL}^{-1}\right)\end{array}$ & $\begin{array}{l}\text { Biomass } \\
\left(\mathrm{g} \mathrm{L}^{-1}\right)\end{array}$ & $\begin{array}{l}\text { Amylase } \\
\text { activity } \\
\left(\mathrm{UmL}^{-1}\right)\end{array}$ & $\begin{array}{l}\text { Extracellular } \\
\text { protein } \\
\left(\mathrm{mg} \mathrm{mL}^{-1}\right)\end{array}$ & $\begin{array}{l}\text { Biomass } \\
\quad\left(\mathrm{g} \mathrm{L}^{-1}\right)\end{array}$ & $\begin{array}{c}\text { Amylase } \\
\text { activity } \\
\left(\mathrm{UmL}^{-1}\right)\end{array}$ & $\begin{array}{l}\text { Extracellular } \\
\text { protein } \\
\left(\mathrm{mg} \mathrm{mL}^{-1}\right)\end{array}$ & $\begin{array}{c}\text { Biomass } \\
\left(\mathrm{g} \mathrm{L}^{-1}\right)\end{array}$ & $\begin{array}{c}\text { Amylase } \\
\text { activity } \\
\left(\mathrm{UmL}^{-1}\right)\end{array}$ \\
\hline INPAR-926 & $1.49 \mathrm{c}$ & $0.90 \mathrm{c}$ & $0.11 \mathrm{c}$ & $3.32 \mathrm{a}$ & $1.70 \mathrm{~d}$ & $0.32 \mathrm{c}$ & $1.84 \mathrm{a}$ & $1.77 \mathrm{~b}$ & $0.42 \mathrm{a}$ \\
\hline INPAR-957 & $1.39 \mathrm{~d}$ & $1.10 \mathrm{bc}$ & $0.16 b$ & $1.50 \mathrm{e}$ & $1.93 \mathrm{~cd}$ & $1.01 \mathrm{a}$ & $1.26 \mathrm{c}$ & $2.03 \mathrm{~b}$ & $0.39 \mathrm{a}$ \\
\hline INPAR-975 & $1.75 b$ & $1.90 \mathrm{a}$ & $0.24 \mathrm{a}$ & $1.67 \mathrm{~d}$ & $2.30 \mathrm{c}$ & $0.51 \mathrm{~b}$ & $1.21 \mathrm{c}$ & $1.93 \mathrm{~b}$ & $0.35 \mathrm{ab}$ \\
\hline INPAR-991 & $1.36 \mathrm{~d}$ & $0.60 \mathrm{~d}$ & $0.26 \mathrm{a}$ & $1.43 \mathrm{e}$ & $1.10 \mathrm{e}$ & $0.54 \mathrm{~b}$ & $1.47 \mathrm{~b}$ & $1.70 \mathrm{~b}$ & $0.30 \mathrm{bc}$ \\
\hline INPAR-988 & $1.73 b$ & $1.20 \mathrm{~b}$ & $0.08 \mathrm{c}$ & $2.16 \mathrm{~b}$ & $3.00 \mathrm{~b}$ & $0.30 \mathrm{c}$ & $1.23 \mathrm{c}$ & $3.07 \mathrm{a}$ & $0.19 \mathrm{~d}$ \\
\hline \multirow[t]{2}{*}{ INPAR-915 } & $2.20 \mathrm{a}$ & $1.30 \mathrm{~b}$ & $0.09 \mathrm{c}$ & $1.84 \mathrm{c}$ & $3.95 \mathrm{a}$ & $0.37 \mathrm{c}$ & $1.81 \mathrm{a}$ & $2.77 \mathrm{a}$ & $0.22 \mathrm{~cd}$ \\
\hline & \multicolumn{3}{|c|}{ Tapioca flour } & \multicolumn{3}{|c|}{ Wheat bran } & \multicolumn{3}{|c|}{ Corn starch } \\
\hline $\begin{array}{l}\text { Rhizobia } \\
\text { strans }\end{array}$ & $\begin{array}{l}\text { Extracellular } \\
\text { protein } \\
\left(\mathrm{mg} \mathrm{mL}^{-1}\right)\end{array}$ & $\begin{array}{l}\text { Biomass } \\
\left(\mathrm{g} \mathrm{L}^{-1}\right)\end{array}$ & $\begin{array}{l}\text { Amylase } \\
\text { activity } \\
\left(\mathrm{UmL}^{-1}\right)\end{array}$ & $\begin{array}{l}\text { Extracellular } \\
\text { protein } \\
\left(\mathrm{mg} \mathrm{mL}^{-1}\right)\end{array}$ & $\begin{array}{c}\text { Biomass } \\
\left(\mathrm{g} \mathrm{L}^{-1}\right)\end{array}$ & $\begin{array}{c}\text { Amylase } \\
\text { activity } \\
\left(\mathrm{UmL}^{-1}\right)\end{array}$ & $\begin{array}{l}\text { Extracellular } \\
\text { protein } \\
\left(\mathrm{mg} \mathrm{mL}^{-1}\right)\end{array}$ & $\begin{array}{c}\text { Biomass } \\
\left(\mathrm{g} \mathrm{L}^{-1}\right)\end{array}$ & $\begin{array}{c}\text { Amylase } \\
\text { activity } \\
\left(\mathrm{UmL}^{-1}\right)\end{array}$ \\
\hline INPAR-926 & $2.52 \mathrm{a}$ & $1.87 \mathrm{~b}$ & $0.17 \mathrm{a}$ & $1.11 \mathrm{~b}$ & $0.90 \mathrm{ab}$ & $0.52 \mathrm{a}$ & $5.70 \mathrm{a}$ & $1.07 \mathrm{c}$ & $0.53 \mathrm{~b}$ \\
\hline INPAR-957 & $2.11 \mathrm{~b}$ & $1.50 \mathrm{~b}$ & $0.21 \mathrm{a}$ & $1.07 \mathrm{bc}$ & $1.00 \mathrm{a}$ & $0.21 \mathrm{~b}$ & $1.61 \mathrm{~d}$ & $1.90 \mathrm{a}$ & $0.23 \mathrm{~d}$ \\
\hline INPAR-975 & $2.17 \mathrm{~b}$ & $2.80 \mathrm{a}$ & $0.22 \mathrm{a}$ & $1.29 \mathrm{a}$ & $0.47 \mathrm{~b}$ & $0.27 \mathrm{~b}$ & $1.50 \mathrm{e}$ & $1.20 \mathrm{bc}$ & $0.35 \mathrm{c}$ \\
\hline INPAR-991 & $2.20 \mathrm{~b}$ & $1.50 \mathrm{~b}$ & $0.22 \mathrm{a}$ & $1.11 \mathrm{~b}$ & $0.83 \mathrm{ab}$ & $0.55 \mathrm{a}$ & $4.25 \mathrm{~b}$ & $0.97 \mathrm{c}$ & $1.59 \mathrm{a}$ \\
\hline INPAR-988 & $1.32 \mathrm{~d}$ & $2.50 \mathrm{a}$ & $0.19 a$ & $1.11 \mathrm{~b}$ & $0.50 \mathrm{ab}$ & $0.27 \mathrm{~b}$ & $3.41 \mathrm{c}$ & $1.17 \mathrm{c}$ & $0.15 \mathrm{de}$ \\
\hline \multirow[t]{2}{*}{ INPAR-915 } & $1.43 \mathrm{c}$ & $0.97 \mathrm{c}$ & $0.20 \mathrm{a}$ & $1.02 \mathrm{c}$ & $0.53 \mathrm{ab}$ & $0.27 b$ & $1.67 \mathrm{~d}$ & $1.70 \mathrm{ab}$ & $0.13 \mathrm{e}$ \\
\hline & \multicolumn{3}{|c|}{ Potato flour } & \multicolumn{3}{|c|}{ Oat flour } & \multicolumn{3}{|c|}{ Maltose } \\
\hline $\begin{array}{l}\text { Rhizobia } \\
\text { strans }\end{array}$ & $\begin{array}{l}\text { Extracellular } \\
\text { protein } \\
\left(\mathrm{mg} \mathrm{mL}^{-1}\right)\end{array}$ & $\begin{array}{c}\text { Biomass } \\
\left(\mathrm{g} \mathrm{L}^{-1}\right)\end{array}$ & $\begin{array}{l}\text { Amylase } \\
\text { activity } \\
\left(\mathrm{UmL}^{-1}\right)\end{array}$ & $\begin{array}{l}\text { Extracellular } \\
\text { protein } \\
\left(\mathrm{mg} \mathrm{mL}^{-1}\right)\end{array}$ & $\begin{array}{c}\text { Biomass } \\
\left(\mathrm{g} \mathrm{L}^{-1}\right)\end{array}$ & $\begin{array}{c}\text { Amylase } \\
\text { activity } \\
\left(\mathrm{UmL}^{-1}\right)\end{array}$ & $\begin{array}{l}\text { Extracellular } \\
\text { protein } \\
\left(\mathrm{mg} \mathrm{mL}^{-1}\right)\end{array}$ & $\begin{array}{c}\text { Biomass } \\
\left(\mathrm{g} \mathrm{L}^{-1}\right)\end{array}$ & $\begin{array}{l}\text { Amylase } \\
\text { activity } \\
\left(\mathrm{UmL}^{-1}\right)\end{array}$ \\
\hline INPAR-926 & $2.51 \mathrm{~b}$ & $1.70 \mathrm{c}$ & $0.24 \mathrm{ab}$ & $2.65 \mathrm{a}$ & $3.00 \mathrm{~d}$ & $0.22 b$ & $1.69 \mathrm{a}$ & $3.20 \mathrm{~b}$ & $1.94 \mathrm{a}$ \\
\hline INPAR-957 & $1.82 \mathrm{~d}$ & $3.63 \mathrm{a}$ & $0.26 \mathrm{ab}$ & $2.43 b$ & $4.20 \mathrm{~b}$ & $0.32 \mathrm{a}$ & $1.54 \mathrm{~b}$ & $2.30 \mathrm{c}$ & $1.78 \mathrm{~b}$ \\
\hline INPAR-975 & $2.10 \mathrm{c}$ & $3.17 \mathrm{ab}$ & $0.25 \mathrm{ab}$ & $2.01 \mathrm{~d}$ & $3.80 \mathrm{~b}$ & $0.21 \mathrm{~b}$ & $1.76 \mathrm{a}$ & $4.13 \mathrm{a}$ & $1.65 \mathrm{c}$ \\
\hline INPAR-991 & $2.10 \mathrm{c}$ & $2.00 \mathrm{c}$ & $0.23 \mathrm{ab}$ & $2.24 \mathrm{c}$ & $5.00 \mathrm{a}$ & $0.16 \mathrm{~b}$ & $1.30 \mathrm{~cd}$ & $1.80 \mathrm{~cd}$ & $1.66 \mathrm{c}$ \\
\hline INPAR-988 & $2.83 \mathrm{a}$ & $1.50 \mathrm{c}$ & $0.21 \mathrm{~b}$ & $2.45 \mathrm{~b}$ & $5.20 \mathrm{a}$ & $0.19 b$ & $1.26 \mathrm{~d}$ & $1.60 \mathrm{de}$ & $1.60 \mathrm{c}$ \\
\hline INPAR-915 & $1.61 \mathrm{e}$ & $2.93 \mathrm{~b}$ & $0.31 \mathrm{a}$ & $1.67 \mathrm{e}$ & $3.63 \mathrm{c}$ & $0.14 b$ & $1.37 \mathrm{c}$ & $1.10 \mathrm{e}$ & $1.63 \mathrm{c}$ \\
\hline
\end{tabular}

Means followed by the same letter are not significantly different at $1 \%$ level.

maltose $(1.94 \mathrm{U})$ and corn starch $(0.53 \mathrm{U})$ media. For the INPA R991, considered in this study as best amylase producer (Fig. 2a), maltose (1.66 U) and corn starch (1.59 U) gave also significant production of extracellular amylase (Table 2). In general, low levels of amylases were registered when mannitol (control) was used as the sole carbon source. In comparison with maltose, the extracellular amylase activity was ten-fold lower (Fig. 2b).

Maltose has been reported to be a better substrate than starch for amylase production by Rhodothermus marinus (12), Clostridium thermosulfurogenes (17), Thermococcus profundus (18), Clostridium sp. (20) and C. thermocellum (38). In contrast, starch was found to be a better substrate than maltose for production of this enzyme by Clostridium thermosulfurogenes (31), Bacillus sp. (33) and B. stearothermophilus (40). Srivastava and Baruah (34) reported that amylase production by $B$. stearothermophilus was better on starch than on maltose. In general, peach palm flour was also revealed to be a good carbon source (Fig. 2b), especially for INPA strain R-957 (Tables 2 and $3)$. Although there are several studies $(1,9,11,12,16,21,29)$ about the use of different grains and tubers for the production of microbial amylases, this is the first report of the use of peach palm flour as a carbon substrate for bacterial amylases. 
Moreover, the results of this essay suggest that several rhizobial amylases could be potentially useful in the saccharification of peach palm starch.

Bacillus species are considered to be the most important sources of microbial amylases (28). In the present study, the average activity of $1.7 \mathrm{U}$ in maltose medium (Fig. 4b) was lower than the values found for Bacillus subtilis (32) and $B$. licheniformis (1), which under optimal culture conditions produced 535 and $252 \mathrm{U}$, respectively; however this value was higher than that reported for B. coagulans and B. circulans (2), Aeromonas sp. and Pseudomonas sp. (36).

The protein content in the culture filtrates varied significantly $(\mathrm{p}<0.01$ ) among strains (Table 3 and Fig. 3a). While INPA strain R-926 exhibited the highest total protein value in this essay, the lowest protein content were observed in the culture filtrates of the INPA strains 915 and R-957 (Fig. 3a). Among the different carbon substrates evaluated, maltose and wheat bran provided the highest and the lowest protein contents, respectively (Fig. $3 b)$. The highest biomass yields were shown by INPA strains R975, R-988 and R-957 (Fig. 4a). As reported previously for
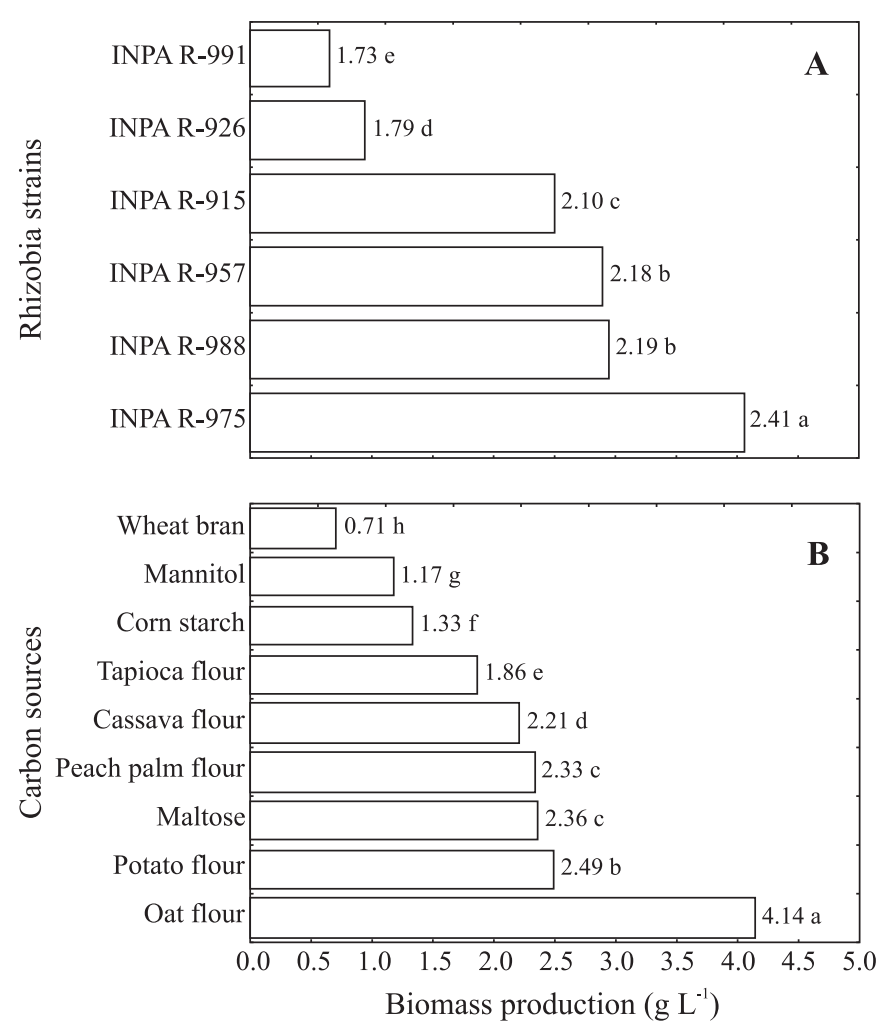

Figure 4. Biomass production of the rhizobia strains evaluated. (A) Averages of all nine carbon sources; (B) Averages of all six rhizobia strains. Means followed by the same letter are not significantly different at $1 \%$ level. extracellular protein, wheat bran did not favour biomass formation of the rhizobial strains studied (Fig. 4b). Thus it is evident that wheat bran as a carbon substrate is not suitable for protein secretion and growth of the rhizobia strains evaluated.

\section{Correlation analysis}

Correlation analysis between rhizobia amylases (averages of all six rhizobia strains) and final $\mathrm{pH}$ of the medium showed a negative relationship for tapioca and potato media, and a significant and positive relationship for wheat bran, corn starch and maltose media (Table 4). In several studies, significant positive associations between final $\mathrm{pH}$ of the medium and microbial amylase production have been reported $(35,37,41)$. The results of this experiment are in agreement with these reports. Significant negative correlation coefficients were observed between rhizobia amylases and extracellular protein for peach palm and potato media, while for oat flour and maltose weak positive correlations were found (Table 4). The negative correlations observed in this study suggest that peach palm and especially potato flour were not specific for rhizobia amylase production, since the secretion of other extracellular enzymes was also stimulated on solid culture media (data not shown). Unsurprisingly, biomass production in the wheat bran, potato flour, oat flour and maltose media was significantly and positively correlated with rhizobia amylases. This type of correlation has also been found by Davis et al. (8) in Bacillus stearothermophilus, Castro et al. (6) in Bacillus amyloliquefaciens and Stamford et al. (35) in Nocardiopsis sp.

On the other hand, rhizobia amylases grown in cassava flour and corn starch were negatively associated with bacterial biomass

Table 4. Pearson product-moment correlation coefficients between amylase activity production and final $\mathrm{pH}$, extracellular protein and biomass of rhizobia strains grown in various media $(\mathrm{n}=18)$.

\begin{tabular}{lccc}
\hline \multirow{2}{*}{$\begin{array}{l}\text { Carbon } \\
\text { substrates }\end{array}$} & \multicolumn{3}{c}{ Variables } \\
\cline { 2 - 4 } & $\begin{array}{c}\text { Final } \\
\mathrm{pH}\end{array}$ & $\begin{array}{c}\text { Extracellular } \\
\text { protein }\end{array}$ & $\begin{array}{c}\text { Biomass } \\
\text { production }\end{array}$ \\
\hline Mannitol & $-0.42^{\mathrm{NS}}$ & $-0.46^{\mathrm{NS}}$ & $0.03^{\mathrm{NS}}$ \\
Peach palm flour & $-0.32^{\mathrm{NS}}$ & $-0.56^{*}$ & $-0.34^{\mathrm{NS}}$ \\
Cassava flour & $-0.29^{\mathrm{NS}}$ & $0.12^{\mathrm{NS}}$ & $-0.84^{* *}$ \\
Tapioca flour & $-0.53^{*}$ & $-0.02^{\mathrm{NS}}$ & $-0.20^{\mathrm{NS}}$ \\
Wheat bran & $0.66^{* *}$ & $0.46^{\mathrm{NS}}$ & $0.56^{*}$ \\
Corn starch & $0.59^{* *}$ & $-0.09^{\mathrm{NS}}$ & $-0.49^{*}$ \\
Potato flour & $-0.52^{*}$ & $-0.86^{* *}$ & $0.56^{*}$ \\
Oat flour & $0.12^{\mathrm{NS}}$ & $0.55^{*}$ & $0.56^{*}$ \\
Maltose & $0.49^{*}$ & $0.47^{*}$ & $0.69^{* *}$ \\
\hline
\end{tabular}

NS $=$ Not significant $*$ and $* *=$ Significant at 5 and $1 \%$, respectively. 
(Table 4). This could be due to presence of inhibitory substances in wheat starch and cassava for the production of amylases, as previously discussed by Haq et al. (16) and Wood (42). Final pH, rhizobial biomass and extracellular protein (averages of all nine growth media) exerted different effects on bacterial amylases (Table 5). Final pH was negatively and positively associated with amylase production for INPA strains R-988 and R-991, respectively. Extracellular protein was also positively correlated with amylase activity in INPA strains R-926, R-988, R-915 and R975. Similarly, bacterial biomass was positively related with amylase production in INPA strain R-926 (Table 5).

The results obtained in the study revealed several Central Amazonian rhizobia strains as promising sources of amylase for biotechnological applications, especially in starch industry.

Table 5. Pearson product-moment correlation coefficients between amylase activity and final $\mathrm{pH}$, extracellular protein and biomass of rhizobia strains (do not considering carbon sources, $\mathrm{n}=27$ ).

\begin{tabular}{|c|c|c|c|}
\hline \multirow{2}{*}{$\begin{array}{l}\text { Carbon } \\
\text { substrates }\end{array}$} & \multicolumn{3}{|c|}{ Variables } \\
\hline & $\begin{array}{c}\text { Final } \\
\mathrm{pH}\end{array}$ & $\begin{array}{c}\text { Extracellular } \\
\text { protein }\end{array}$ & $\begin{array}{l}\text { Biomass } \\
\text { production }\end{array}$ \\
\hline INPAR-926 & $0.32^{\mathrm{NS}}$ & $0.45^{*}$ & $0.53^{* *}$ \\
\hline INPAR-988 & $-0.78^{* *}$ & $0.43^{*}$ & $-0.14^{\mathrm{NS}}$ \\
\hline INPAR-957 & $0.12^{\mathrm{NS}}$ & $0.25^{\mathrm{NS}}$ & $0.52^{* *}$ \\
\hline INPAR-991 & $0.54^{* *}$ & $0.02^{\mathrm{NS}}$ & $-0.27^{\mathrm{NS}}$ \\
\hline INPAR-915 & $0.27^{\mathrm{NS}}$ & $0.39^{*}$ & $-0.23^{\mathrm{NS}}$ \\
\hline INPAR-975 & $-0.37^{\mathrm{NS}}$ & $0.52^{* *}$ & $0.01^{\mathrm{NS}}$ \\
\hline
\end{tabular}

NS $=$ Not significant $*$ and $* *=$ Significant at 5 and $1 \%$, respectively.

\section{ACKNOWLEDGEMENTS}

The authors gratefully acknowledge financial support from Fundação de Amparo à Pesquisa do Estado do Amazonas (FAPEAM Process 624/04) and Conselho Nacional de Desenvolvimento Científico e Tecnológico (CNPq Process 474007/2003-0). The author LAO is a CNPq fellow.

We would like to thank Dr. Charles Roland Clement (INPA/ CPCA) for reading and revising the English text.

\section{RESUMO}

\section{Produção de amilase por rizóbios, usando várias substâncias amiláceas como substratos de carbono}

Seis isolados de rizobia nativos da Amazonia Central foram selecionados para a produção de amilases em meio de cultura líquido, usando várias substâncias amiláceas como fontes de carbono. Os resultados mostraram que todos os isolados de rizobia podem produzir quantidades diferenciadas de proteína extracelular, biomassa e amilases com diferentes tipos de fontes de carbono. Entre as fontes testadas, maltose foi a melhor para as produções de proteína e amilase. Por outro lado, a produção de biomassa pelos isolados de rizobia foi maior na presença de farinha de aveia. O isolado INPA R-926 mostrou-se um bom produtor de amilase nos meios com maltose (1,94 U) e amido de milho (0,53 U). O isolado INPA R-991 também foi um bom produtor de amilase com rendimentos significativos na presença de maltose (1,66 U) e amido de milho (1,59 U). As análises de correlação revelaram relações positivas e significativas entre as atividades de amilase e $\mathrm{pH}$ final $(\mathrm{r}=0,49, \mathrm{P}<0,05)$, proteína extracelular $(r=0,47, \mathrm{P}<0,47)$ e a produção de biomassa $(\mathrm{r}=$ $0,69, \mathrm{P}<0,01)$ no meio com maltose. Os resultados obtidos nesse estudo revelaram alguns isolados de rizóbio da Amazônia Central como fontes promissoras de amilases para aplicações biotecnológicas, especialmente na indústria do amido.

Palavras-chave: Rhizobium, Bradyrhizobium, feijão caupi, soja, farinha de pupunha, Amazônia Central

\section{REFERENCES}

1. Aiyer, P.V.D. (2004). Effect of C:N ratio on alpha amylase production by Bacillus licheniformis SPT 27. Afr. J. Biotechnol., 3, 519-522.

2. Ajayi, A.O.; Fagade, O.E. (2003). Utilization of corn starch as substrate for $\beta$-amylase by Bacillus spp. Afr. J. Biomed. Res., 6, 3742 .

3. Barnet, Y.M.; Catt, P.C. (1991). Distribution and characteristics of root-nodule bacteria isolated from Australia Acacia spp. Plant Soil., $135,109-120$

4. Boddey, L.H.; Hungria, M. (1997). Phenotypic grouping of Brazilian Bradyrhizobium strains which nodulate soybean. Biol. Fertil. Soils. 25, 407-415.

5. Buzzini, P.; Martini, A. (2002). Extracellular enzymatic activity profiles in yeast and yeast-like strains isolated from tropical environments. J. Appl. Microbiol., 93, 1020-1025.

6. Castro, G.R.; Mendez, B.S.; Siñeriz, F. (1993). Amylolytic enzymes produced by Bacillus amyloliquefaciens MIR-41 in bath and continuous culture. J. Chem. Technol., 50, 289-294.

7. Crueger, W.; Crueger, A. (1989). Industrial Microbiology. Sinauer Associates, Sunderland, MA.

8. Davis, P.E.; Cohen, D.L.; Whitaker, A. (1980). The production of alpha amylase in batch and chemostatic culture. Antonie van Leenwenhoek., 46, 391-398.

9. Fossi, B.T.; Tavea, F.; Ndjouenkeu, R. (2005). Production and partial characterization of a thermostable amylase from ascomycetes yeast strain isolated from starchy soils. Afr. J. Biotechnol., 4, 14-18.

10. Ghasemi, M.F.; Bakhtiari, M.R.; Fallahpour, M.; Noohi, A.; Moazami, N.; Amidi, Z. (2004). Screening of Urease Production by Aspergillus niger strains. Iran Biomed. J., 8, 47-50.

11. Ghosh, S.B.; Chandra, A.K. (1984). Nutritional requirements and cultural characteristics of Bacillus apiarius CBML-152 for the production of thermostable alpha amylase. Zbl. Mikrobiol., 139, 293-304.

12. Gomes, I.; Gomes, J.; Steiner, W. (2003). Highly thermostable amylase and pullulanase of the extreme thermophilic eubacterium 
Rhodothermus marinus: production and partial characterization. Biores. Technol., 90, 207-214.

13. Gornall, A.G.; Bardawill, C.J.; David, M.M. (1949). Determination of serum proteins by mean of biuret reaction. J. Biol. Chem., 177, 751-756.

14. Gupta, R.; Gigras, P.; Mohapatra, H.; Goswami, V.K.; Chauhan, B. (2003). Microbial $\alpha$-amylases: a biotechnological perspective. Process Biotechem., 38, 1599-1616.

15. Haq, I.; Ashraf, H.; Omar, S.; Qadeer, M.A. (2002). Biosynthesis of Amyloglucosidase by Aspergillus niger Using Wheat Bran as Substrate. Pak. J. Biol. Sci., 5, 962-964.

16. Haq, I.; Ashraf, H.; Qadeer, M.A.; Javed, I. (2005). Pearl millet, a source of alpha amylase production by Bacillus licheniformis. Biores. Technol., 96, 1201-1204.

17. Hyun, H.H.; Zeikus, J.C. (1985). General biochemical characterization of thermostable pullulanase and glucoamylase from Clostridium thermohydrosulfuricum. Appl. Environ. Microbiol., 49, 1168-1173.

18. Kwak, Y.S.; Akiba, T.; Kudo, T. (1998). Purification and characterization of $\alpha$-amylase from hyperthermophilic archaeon Thermococcus profundus, which hydrolyzes both $\alpha-1,4$ and $\alpha-1,6$ glucosidic linkages. J. Ferment. Bioeng., 86, 363-367.

19. Jordan, D.C. (1984). Genera Rhizobium and Bradyrhizobium. In: Krieg, N.T.; Holt, J.G. (eds). Bergey's Manual of Systematic Bacteriology. Williams \& Wilkins, Baltimore, USA, p.235-244.

20. Madi, E.; Antranikian, G.; Ohmiya, K.; Gottschalk, G. (1987). Thermostable Amylolytic Enzymes from a New Clostridium Isolate. Appl Environ Microbiol., 53, 1661-1667.

21. Mahmoud, S.A.; Abdel-Hafez, A.M.; Mashhoor, W.A. Refaat, A.A. (1978). Utilization of industrial and agricultural by-products for fungal amylase production. Zentralbl Bakteriol Naturwiss, 133, 115120.

22. Minamisawa, T.; Onodera, S.; Kubota, M.; Asami, T. (1992). Genetic relatedness of Bradyrhizobium japonicum field isolates as revealed by repeated sequences and various other characteristics. Appl. Environ. Microbiol., 58, 2832-2839.

23. Mohamed, S.H.; Smouni, A.; Neyra, M.; Kharchaf, D.; Filali-Maltouf, A. (2000). Phenotypic characteristics of root-nodulatin bacteria isolated from Acacia spp. grown in Libya. Plant Soil., 224, 171183.

24. Moreira, F.M.S.; Gillis, M.; Pot, B.; Kerskers, K.; Franco, A.A. (1993). Characterization of rhizobia isolated from different divergence groups of tropical leguminosae by comparative polyacrylamide gel eletrophoresis of their total proteins. Syst. Appl. Microbiol., 17, 135-146.

25. Nelson, N. (1944). A photometric adaptation of the Somogyi method for the determination of glucose. J. Biol. Chem., 153, 375-380.

26. Nigam, P.; Singh, D. (1995). Enzymes and microbial system involved in starch processing. Enzyme Microbiol. Technol., 17, 770-778.

27. Odee, D.W.; Sutherland, J.M.; Makatiani, E.T.; McInroy, S.G.; Sprent, J.I. (1997) Phenotypic characteristics and composition of rhizobia associated with woody legumes growing in diverse Kenyan conditions. Plant Soil., 188, 65-75.

28. Pandey, A.; Nigam, P.; Soccol, C.R.; Soccol, V.T.; Singh, D.; Mohan, R. (2000). Advances in microbial amylases. Biotechnol. Appl. Biochem., 31, 135-152.

29. Ramesh, B.; Reddy, P.R.M.; Seenayya, G.; Reddy, G. (2001). Effect of various flours on the production of thermostable $\beta$-amylase and pullulanase by Clostridium thermosulfurogenes SV2. Biores. Technol., 76, 169-171.

30. Rao, M.B.; Tanksale, A.M.; Gathe, M.S.; Deshpande, V.V. (1998). Molecular and biotechnological aspects of microbial proteases. Microbiol. Mol. Biol. Rev., 62, 597-635.

31. Reddy, P.R.M.; Reddy, G.; Seenayya, G. (1999). Enhanced production of thermostable $\beta$-amylase and pullulunase in the presence of surfactants by Clostridium thermosulfurogenes SV2. Process Biochem., 34, 87-92.

32. Riaz, N.; Haq, I.; Qadeer, M.A. (2003). Characterization of $\alpha$-amylase by Bacillus subtilis. Int. J. Agr. Biol., 5, 249-252.

33. Santos, E.O.; Martins, M.L.L. (2003). Effect of the medium composition on formation of amylase by Bacillus sp. Braz. Arch. Biol. Technol., 46, 129-134.

34. Srivastava, R.A.C.; Baruah, J.N. (1986). Culture conditions for production of thermostable amylase Bacillus stearothermophilus. Appl. Environ. Microbiol., 52, 179-184.

35. Stamford, T.L.M.; Stamford, N.P.; Coelho, L.C.B.B.; Araújo, J.M. (2001). Production and characterization of a thermostable $\alpha$-amylase from Nocardiopsis sp. endophyte of yam bean. Biores. Technol., 76, 137-141.

36. Sugita, H.; Kawasaki, J.; Deguchi Y. (1997). Production of amylase by the intestinal microflora in cultured freshwater fish. Lett. Appl. Microbiol., 24, 105-108.

37. Sunna, A.; Hashwa, F. (1990). Thermostable amylase from an aerobic Gram-negative, nonspore forming thermophilic bacteria. Biotechnol. Lett., 12, 433-438.

38. Swamy, M.V.; Sai, R.M.; Seenayya, G. (1994). $\beta$-amylase from Clostridium thermocellum SSA thermophilic anaerobic cellulolytic bacterium. Lett. Appl. Microbiol., 18, 301-304.

39. Vincent, J.M. (1970). A manual for the practical study of rootnodules bacteria. Blackwell Science Publication, Oxford, UK.

40. Wind, R.D.; Buitelaar, R.M.; Eggink, G.; Huizing, H.J.; Dijkhuizen, L. (1994). Characterization of a new Bacillus stearothermophilus isolate: a highly thermostable $\alpha$-amylase producing strain. Appl. Microbiol. Biotechnol., 41, 155-162.

41. Wijbenga, D.J.; Beldman, G.; Veen, A.; Binnema, D.L. (1991). Production of native-starch-degrading enzymes by a Bacillus firmus/ lentus strain. Appl. Microbiol. Biotechnol., 35, 180-184.

42. Wood, T. (1965). The cyanogenic glucosides content of cassava products. J. Science Food Agric., 16, 300-305.

43. Young, J.P.W.; Haukka, K.E. (1996). Diversity and phylogeny of rhizobia. New Phytol., 133, 87-94. 